\title{
The Synthesis Temperature Selection of Cerium Doped Lutetium Yttrium Oxyorthosilicate Single Crystal Powder
}

\author{
Wenping Zhou ${ }^{1,2, a}$, Zhixin Ma ${ }^{2}$, Yunping Cai ${ }^{1}$, Xiaoguo $\mathrm{Bi}^{1}$, Tianxiao $\mathrm{Li}^{1}$, Wei \\ Niu ${ }^{1}$, Xudong Sun ${ }^{2}$, Qikang Lu ${ }^{1}$
}

1. School of Energy and Power, Shenyang Institute of Engineering, Shenyang, Liaoning,110136,China

2. School of Materials Science and Engineering, Northeastern University, Shenyang,

Liaoning,110004,China

zhouwp@sie.edu.cn Keywords: Cerium doped lutetium yttrium oxyorthosilicate single crystal( $\mathrm{Ce}$ : $\mathrm{Lu}_{2} \mathrm{Y}_{2} \mathrm{SiO}_{5}$ ); Flame
fusion method; SEM analysis; XRD analysis; SEM analysis; XRD analysis

\begin{abstract}
Flame fusion preparation of LYSO: Ce single crystal need excellent mobility raw powder of high purity. The solid phase synthesis of LYSO:Ce single crystal powder of high quality is prepared. After removal of moisture, weighing and grinding, the original high purity powder sintering experiments are conducted under $1100{ }^{\circ} \mathrm{C}, 1200{ }^{\circ} \mathrm{C}, 1300{ }^{\circ} \mathrm{C}, 1400{ }^{\circ} \mathrm{C}$ and $1500{ }^{\circ} \mathrm{C}$ respectively. The SEM analysis and XRD results showed that it can obtain high purity powder with good fluidity at $1400^{\circ} \mathrm{C}$ and $1500^{\circ} \mathrm{C}$ conditions.
\end{abstract}

\section{Introduction}

Because of its high light yield $(\sim 30000 \mathrm{ph} / \mathrm{MeV})$ and energy resolution $(\sim 10 \%)$ and the delay time of fast ( 40ns), Cerium doped lutetium yttrium oxyorthosilicate single crystal (LYSO:Ce) single crystal has become the most valuable materials of the field of nuclear medicine imaging, high-energy physics experiment[1 4]. As everyone knows, the basic requirements for the crystal growth of powder is high purity, small particle size and good liquidity while the flame fusion growth of LYSO:Ce crystal. But the synthesis power of lutetium yttrium oxyorthosilicate (LYSO:Ce) single crystal includes many components about difference density and so, to meet the requirements of the synthesis of the powder is relatively difficult. One of the main factors is that to control the synthesis temperature. This paper will use the solid phase synthesis method to preparation powder for flame fusion growth of LYSO:Ce single crystal.

\section{Preparation for Experiment}

The purity of raw materials used is $99.99 \%$ of high purity $\mathrm{Lu}_{2} \mathrm{O}_{3}, \mathrm{Y}_{2} \mathrm{O}_{3}, \mathrm{SiO}_{2}$ and $\mathrm{CeNO}_{3}$. In the composition of raw material, in order to avoid the disadvantages of quadrivalence cerium reduction, this experiment chooses the trivalent cerium - CeNO3.

In order to remove moisture, the raw materials are preheated in the crucible respectively for 700 hours and kept for 8 hours. According to the calculation of molar ratio of raw materials, the quality percentage is $80.39 \%$ for $\mathrm{Lu}_{2} \mathrm{O}_{3}, 5.07 \%$ for $\mathrm{Y}_{2} \mathrm{O}_{3}, 13.56 \%$ for $\mathrm{SiO}_{2}, 0.98 \%$ for $\mathrm{CeNO}_{3}$. After weighing precisely, all power is mixed in a mortar and grinded, then to prepared for sintering.

\section{Experiment and Analysis}

The powders are sintered at $1100^{\circ} \mathrm{C}, 1200^{\circ} \mathrm{C}, 1300^{\circ} \mathrm{C}, 1400^{\circ} \mathrm{C}$ and $1500^{\circ} \mathrm{C}$ respectively for eight hours. The scanning electron microscopy (SEM) analysis is used to analysis Ingredients and X-ray diffraction analysis (XRD) is used to analyze the phase [5-9].

SEM analysis As shown in Figure 1-5 respectively under the SEM for LYSO:Ce powder after eight hours at $1100^{\circ} \mathrm{C}, 1200^{\circ} \mathrm{C}, 1300^{\circ} \mathrm{C}, 1400^{\circ} \mathrm{C}$ and $1500^{\circ} \mathrm{C}$, it can be seen from the figures, Fist, when the powder is sinteringed in the solid phase synthesis of $1100^{\circ} \mathrm{C}$, the reaction takes place only in a small amount of powder. Most of the powder is still in the reunion state. Second, when the synthesis temperature increases to 1200 degrees, most of the powder began to react, nucleate and 
growth. Combined with phase analysis, there has been no raw material powder composition. Third, when the synthesis temperature increases to $1300^{\circ} \mathrm{C}$, powder sintering gradually and be shaped. The forth, when the temperature is to 1400 DEG, powder has been nucleation and growth which can be observed in the neck phenomenon. Combined with phase analysis, we can deduce 1400 degrees in the synthesis temperature, can be used to prepared pure LYSO:Ce powder. The last, to further improve the synthesis temperature reach 1500 degrees, the grains grow further, and sinter together, meanwhile, the flowability of the powders is greatly improved from macroscopic observation.

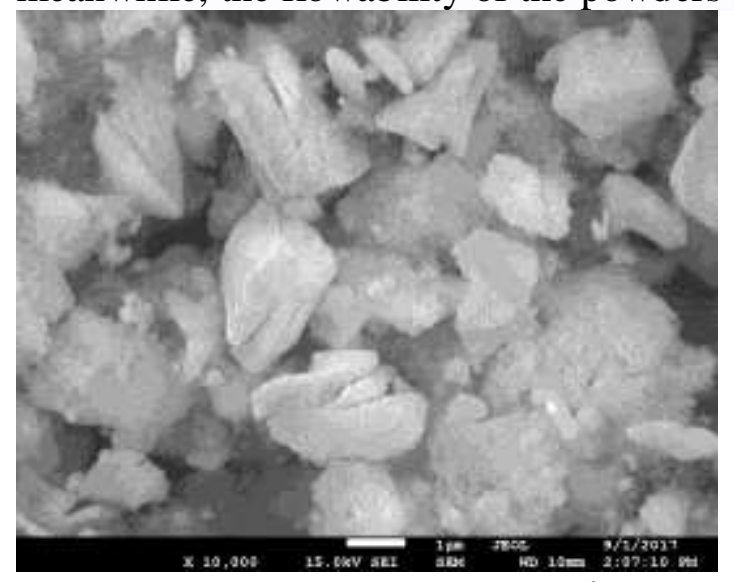

Figure 1. Analysis of powerd at $1100^{\circ} \mathrm{C}$ by SEM

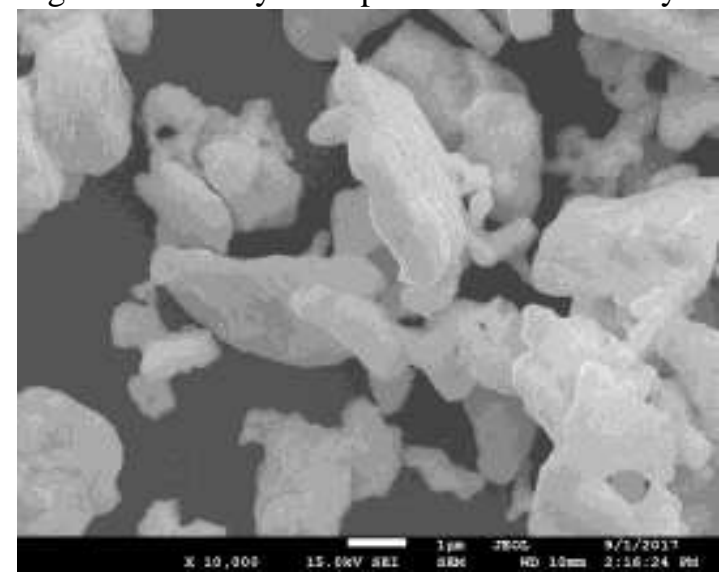

Figure 3. Analysis of powerd at $1300^{\circ} \mathrm{C}$ by SEM

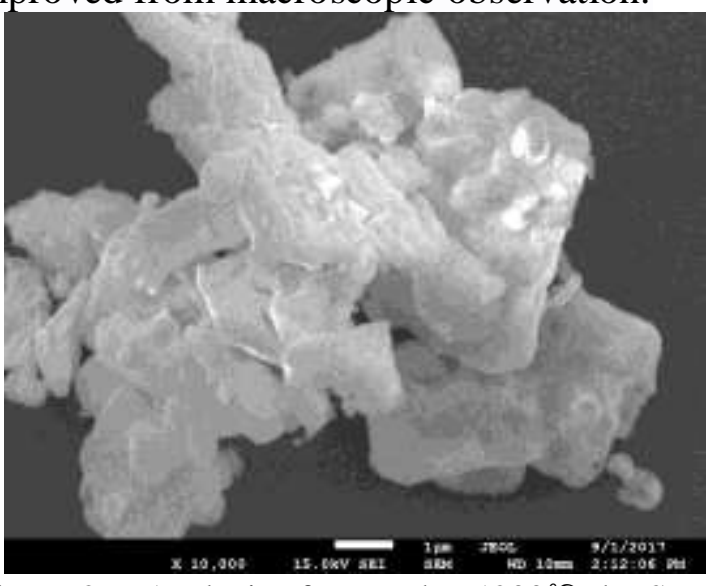

Figure 2. Analysis of powerd at $1200^{\circ} \mathrm{C}$ by SEM

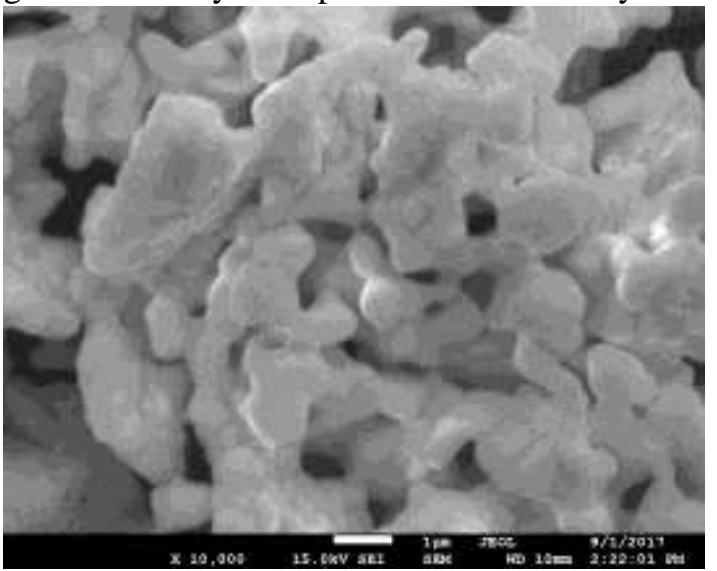

Figure 4. Analy sis of powerd at $1400^{\circ} \mathrm{C}$ by SEM

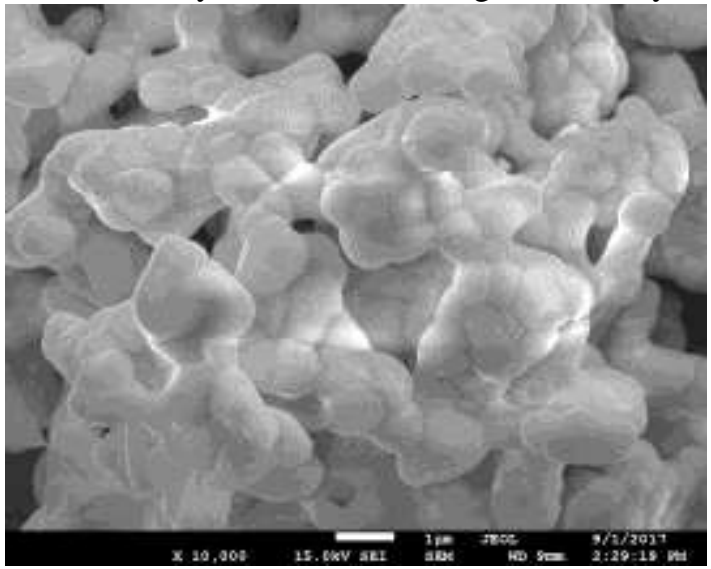

Figure5. Analysis of powerd at $1500^{\circ} \mathrm{C}$ by SEM

XRD analysis In order to examine whether other phases exist in the crystal, we make LYSO:Ce powder synthesized at $1100^{\circ} \mathrm{C}, 1200^{\circ} \mathrm{C}, 1300^{\circ} \mathrm{C}, 1400^{\circ} \mathrm{C}$ and $1500^{\circ} \mathrm{C}$ to solid phase analysis by XRD detection. The results are shown in figures 6-10. It can be seen from the figures: First, when the powder at $1100^{\circ} \mathrm{C}$ condition, in a large part of lutetium oxide powder has no solid phase reaction, and only a small amount of LYSO:Ce phase has been detected by phase detection. IT is proved that the reaction temperature is too low. Second, when the powder at $1200^{\circ} \mathrm{C}$ or 
$1300^{\circ} \mathrm{C}$ condition, pyrosilicate phase appears, and with the increase of reaction temperature, pyrosilicate phase gradually reduced. Third, when the synthesis temperature is to $1400^{\circ} \mathrm{C}$ and $1500^{\circ} \mathrm{C}$, pyrosilicate phase gradually disappeared, pure LYSO:Ce phase has been synthesized..

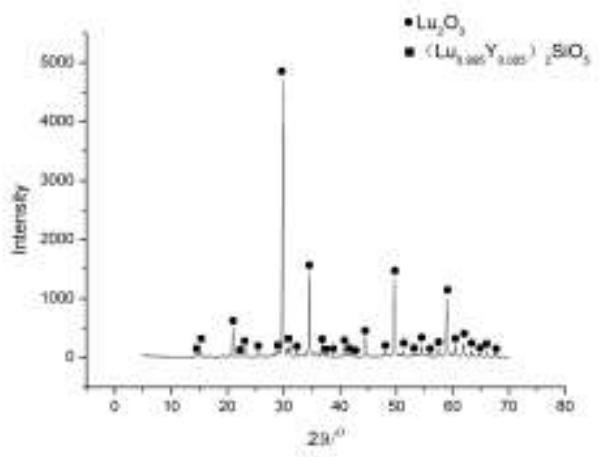

Figure 6. LYSO:Ce powder under $1100^{\circ} \mathrm{C}$ at XRD

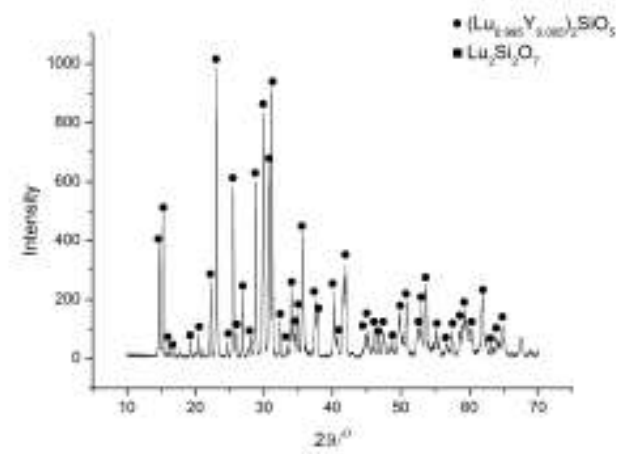

Figure8. LYSO:Ce powder under $1300^{\circ} \mathrm{C}$ at XRD

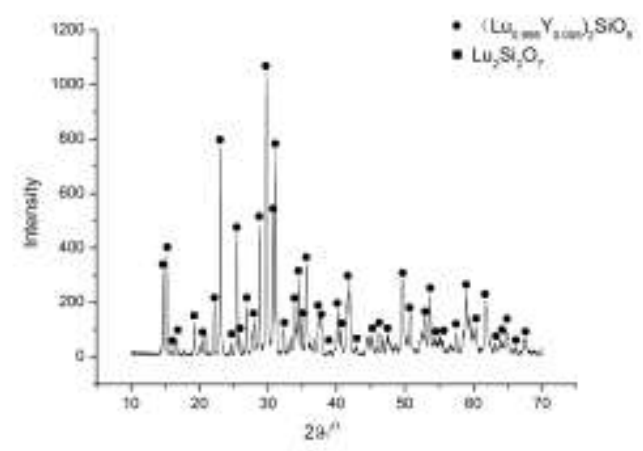

Figure 7. LYSO:Ce powder under $1200^{\circ} \mathrm{C}$ at XRD

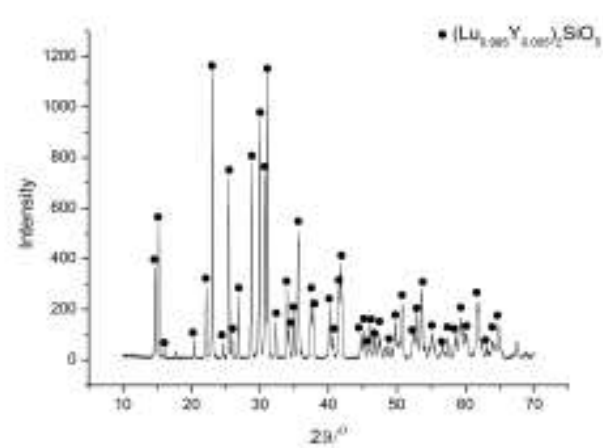

Figure 9. LYSO:Ce powder under $1400^{\circ} \mathrm{C}$ at XRD

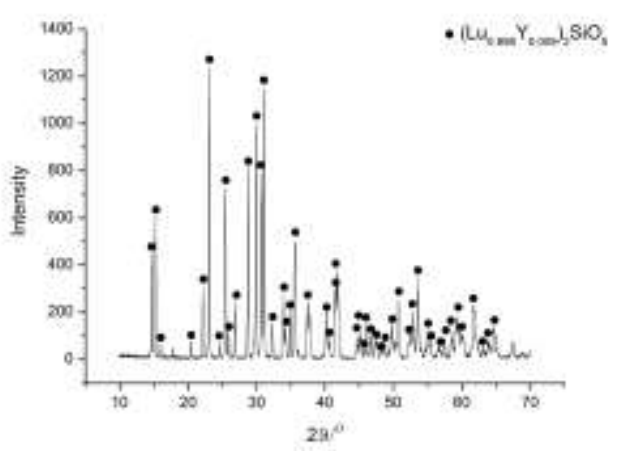

Figure 10. LYSO:Ce powder under $1500^{\circ} \mathrm{C}$ at XRD

In order to see the LYSO: Ce powder purity more clearly, we do comparative analysis of XRD map of LYSO:Ce and LSO:Ce standard card, as shown in figure 11. 

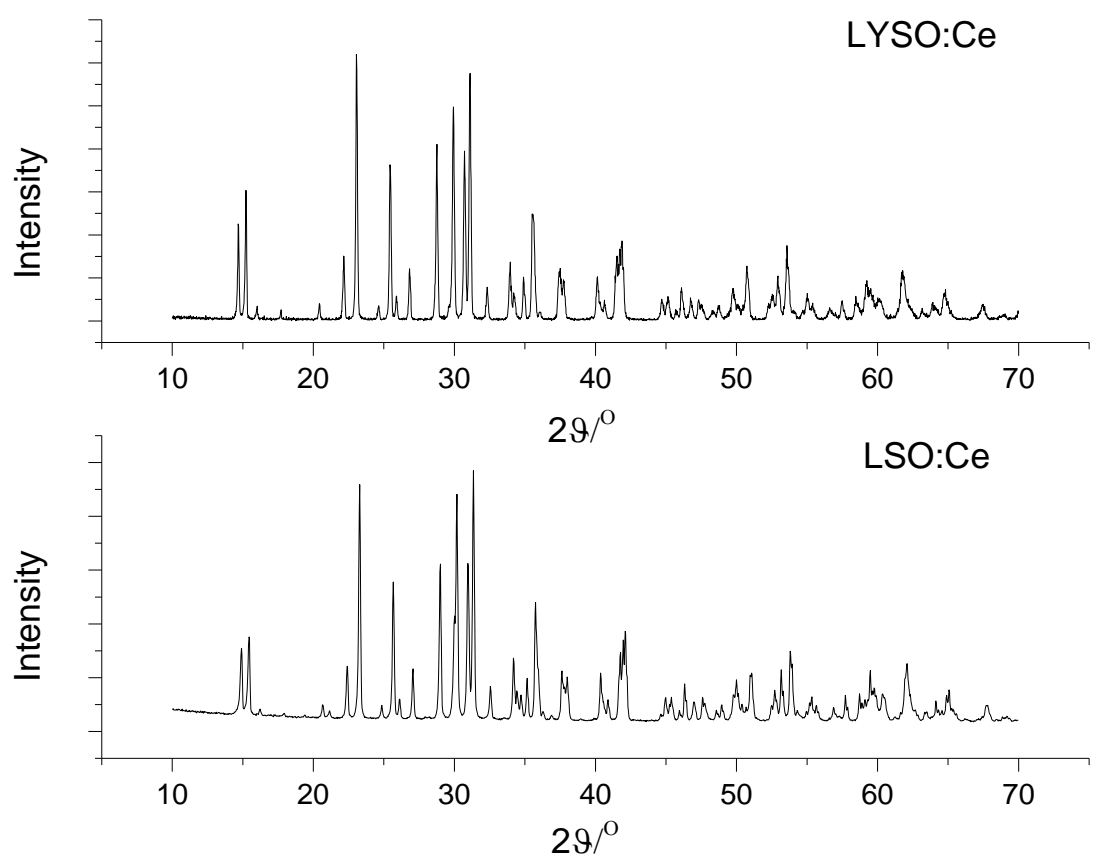

Figure 11. LYSO: Ce and LSO powder in XRD contrast map for $1500^{\circ} \mathrm{Cafter} 8 \mathrm{~h}$ sintering

It can be seen from the figure11, the lyso diffraction contrast of LYSO: Ce with LSO: Ce maps , the peaks left shift with a certain degree, and this is due to the incorporation of the yttrium. According to the Prague equation: $2 \mathrm{~d} \sin \theta=\mathrm{n} \lambda$, because the greater atomic radius of yttrium (2.27 $\AA$ ) than the atomic radius of lutetium $(2.25 \AA)$, d value increases after the incorporation of yttrium and theta value decreases, which result in the diffraction peaks of LYSO: Ce left shift in some degree.

\section{Conclusion}

The SEM and XRD phase analysis for solid phase synthesis of LYSO: Ce powder is done and solid phase synthesis at $1400^{\circ} \mathrm{C}$ can be prepared with high purity LYSO: Ce powder with the increase of powder synthesis temperature, we also can improve the macro liquidity powder. But the temperature is recommended below $1500^{\circ} \mathrm{C}$, so as not to affect the other key factors like powder fluidity.

\section{References}

[1]Pidol L, Kahn-Harari, A ;Viana, B et al. SCINTILLATION DETECTORS-High Efficiency of Lutetium Silicate Scintillators, Ce-Doped LPS, and LYSO[J]. IEEE Transactions on Nuclear Science, 2004, 51 (3): 1084-1087.

[2]Valais, IG; Michail, CM; David, SL et al. Luminescence emission properties of ( $\mathrm{Lu}, \mathrm{Y})(2) \mathrm{SiO}_{5}$ : $\mathrm{Ce}(\mathrm{LYSO}: \mathrm{Ce})$ and $(\mathrm{Lu}, \mathrm{Y}) \mathrm{AlO}_{3}$ : Ce (LuYAP : Ce) single crystal scintillators under medical imaging conditions, IEEE T NUCL SCI , 55 (2) 785-789.

[3]T. Kimble, M. Chou, and B. H. T. Chai et al. Scintillation properties of Ce:LYSO crystals[J].IEEE Nuclear Science Symp. Conf, 2002, 3: 1434-1437.

[4]W.Chewpraditkul, L.Swiderski, M.Moszynski, et al. Scintillation properties of LuAG:Ce, YAG:Ce for gamma-ray detection. IEEE Trans. Nucl. Sci. 2009, 56, 3800-3805.

[5] Zhang Ke, Zhang Lehui, science and technology of crystal growth (Second Edition) volumes, Science Press, 1997.5:251-299

[6] 《Crystal Growth Technology》, John Wiley, Hans J.Scheel and Tsuguo Fukuda, 2003.

[7]Crystal Growth for Bginners: fundamentals of nucleation, crystal growth and epitaxy, World 
Scientific, I.V.Markov1998

[8] Yao Lianzeng, "Fundamentals of crystal growth", University of Science \& Technology China press, 1995

[9] min Zhao, physics foundation of crystal growth, science and Technology Press, 1982 